\title{
Spin Incommensurability and Two Phase Competition in Cobaltites
}

\author{
D. Phelan, ${ }^{1,2}$ Despina Louca, ${ }^{1, *}$ K. Kamazawa, ${ }^{1}$ S.-H. Lee, ${ }^{1}$ S. N. Ancona, ${ }^{3}$ S. Rosenkranz, ${ }^{3}$ Y. Motome,${ }^{4}$ M. F. Hundley, ${ }^{5}$ \\ J. F. Mitchell, ${ }^{3}$ and Y. Moritomo ${ }^{6}$ \\ ${ }^{1}$ Department of Physics, University of Virginia, Charlottesville, Virginia 22904, USA \\ ${ }^{2}$ NIST Center for Neutron Research, Gaithersburg, Maryland 20899, USA \\ ${ }^{3}$ Materials Science Division, Argonne National Laboratory, Argonne, Illinois 60439, USA \\ ${ }^{4}$ Department of Applied Physics, University of Tokyo, Tokyo 113-8656, Japan \\ ${ }^{5}$ Los Alamos National Laboratory, Los Alamos, New Mexico 87454, USA \\ ${ }^{6}$ Department of Physics, University of Tsukuba, Tsukuba 305-8577, Japan
}

(Received 24 August 2006; published 6 December 2006)

\begin{abstract}
The perovskite $\mathrm{LaCoO}_{3}$ evolves from a nonmagnetic Mott insulator to a spin cluster ferromagnet (FM) with the substitution of $\mathrm{Sr}^{2+}$ for $\mathrm{La}^{3+}$ in $\mathrm{La}_{1-x} \mathrm{Sr}_{x} \mathrm{CoO}_{3}$. The clusters increase in size and number with $x$ and the charge percolation through the clusters leads to a metallic state. Using elastic neutron scattering on $\mathrm{La}_{1-x} \mathrm{Sr}_{x} \mathrm{CoO}_{3}$ single crystals, we show that an incommensurate spin superstructure coexists with the FM spin clusters. The incommensurability increases continuously with $x$, with the intensity rising in the insulating phase and dropping in the metallic phase as it directly competes with the commensurate FM, itinerant clusters. The spin incommensurability arises from local order of $\mathrm{Co}^{3+}-\mathrm{Co}^{4+}$ clusters but no longrange static or dynamic spin stripes develop. The coexistence and competition of the two magnetic phases explain the residual resistivity at low temperatures in samples with metalliclike transport.
\end{abstract}

DOI: 10.1103/PhysRevLett.97.235501

PACS numbers: $61.12 .-\mathrm{q}, 71.30 .+\mathrm{h}, 71.70 .-\mathrm{d}$

Competing interactions and electron localization are at the heart of the complex phenomena prevailing in transition metal oxides. The manganites and cuprates are two examples in which spin, charge, lattice, and orbital degrees of freedom conspire in ways that create unconventional states. Such states emerge when holes are doped in the antiferromagnetic (AFM) ground state of the parent compound. In the case of manganites that exhibit a fieldinduced colossal magnetoresistance (CMR) [1], the competition between the tendency for strong localization via polaron formation and metallic itineracy leads to phase separation and charge or spin stripe formation [2]. In the cuprates and their surrogates, the nickelates [3], comparable localization schemes involving stripes have been described that are key towards our understanding of the 20-year-old problem of high- $T_{C}$ superconductivity. Although the intricate electronic correlations that give rise to inhomogeneous states have been uniquely discussed in the context of doped AFM Mott insulators, the present work focuses on cobaltites with the perovskite structure whose parent compound is a nonmagnetic insulator that becomes magnetically ordered only when holes are doped. Even though this system is distinct from the manganites and cuprates with unique features, some of which will be described below, the presence of a magnetic inhomogeneity in a seemingly ordered state is evident here as well, and may provide important insight to the formation of stripes. The pinning of stripes into statically ordered arrays results in insulating states as in manganites and nickelates, while dynamic stripes are anticipated in the superconducting cuprates. The presence of intermediate states between the two extremes would be useful in understanding the tran- sition from one state to the other, and the cobaltites are ideally suited in this effort.

The cobaltites exhibit a range of properties including superconductivity [4], thermoelectricity [5], ionic conductivity [6], magnetic and insulator-metal transitions (IMT), and magnetoresistivity. Of particular relevance is the influence of the electronic transitions of the $\mathrm{Co}^{3+}$ ion $\left(3 d^{6}\right)$ from the nonmagnetic ground state into excited states [7] on the properties [8-11]. But even when the $\mathrm{Co}^{3+}$ ions become thermally activated in the parent compound, $\mathrm{LaCoO}_{3}$, no static magnetic ordering ensues. Only the mixing of $\mathrm{Co}^{3+}$ with $\mathrm{Co}^{4+}$ ions, the latter introduced by adding charges into the parent lattice, leads to a percolative phase transition of a magnetically ordered and metallic state $[10,11]$ that is mediated by double exchange coupling [12]. In spite of the good conducting properties at low temperatures, however, the CMR response remains mysteriously suppressed with about a $10 \%$ increase in a $9 \mathrm{~T}$ field compared to a $10^{6} \%$ increase in some manganites. In the present study, we examine the self-organization of an incommensurate magnetic phase and its role in destabilizing the ferromagnetic (FM) metallic state and rendering the resistivity response observed at low temperatures. Our results suggest that the particular nature of cobaltites arises from the competition between magnetic localization and charge itineracy resulting in the formation of stripelike structures.

The observations were made by cold inelastic neutron scattering. The experiment revealed a new effect in $\mathrm{La}_{1-x} \mathrm{Sr}_{x} \mathrm{CoO}_{3}$, in which spin superstructures form at temperatures lower than the macroscopic transition temperatures. The onset temperature and the ordering wave vector 
vary continuously with the nominal charge concentration while the latter is incommensurate with the lattice periodicity. The first spin ordering leading to the macroscopic transition is charge driven aided by the double exchange interaction of $\mathrm{Co}^{3+}$ and $\mathrm{Co}^{4+}$, while the second ordering is spin driven where no macroscopic transition is observed [13]. It is suggested that the second transition may be stimulated by phase segregation where charge carriers separate into hole-rich and hole-poor regions [14]. The appearance of the short-range spin superstructure upon cooling might contribute to the effects that give rise to the residual resistivity particularly in the metallic state. This behavior is reminiscent of the ordering effects observed in manganites and cuprates. In the manganites, tendencies for localization lead to an insulating state [15] accompanied by charge and orbital ordering, while in the cuprates or nickelates such behavior leads to the formation of stripes [16]. The short-range spatial extension of the spin "stripes" observed in cobaltites signals the competition between two mechanisms that render the system in a frustratedlike state.

The single crystals (the same as reported in Ref. [10]) were grown using the floating zone technique. The neutron measurements were carried out at SPINS, the cold neutron triple-axis spectrometer located at the NIST Center for Neutron Research. Neutron scattering was used as it provides insight into the magnetic correlations within a crystal. The neutron is a spin- $\frac{1}{2}$ particle that interacts with the spins in the crystal via the magnetic dipole interaction, yielding a magnetic component to the scattering crosssection that is proportional to the spin-spin correlation function, $\left\langle S_{R}(t) S_{R^{\prime}}(0)\right\rangle$. The elastic scattering, indicative of static correlations in the time scale of the neutron experiment, was measured in the $(h h l)$ plane for crystals of $\mathrm{La}_{1-x} \mathrm{Sr}_{x} \mathrm{CoO}_{3}$, and the scattering contour at $\sim 8 \mathrm{~K}$ is shown in Fig. 1(a) for the $x=0.15$ (similar measurements were performed on $x=0.1$ and 0.2 but are not shown). The prominent feature in the center of the contour map is the isotropic scattering centered around the (001) Bragg peak. Also apparent in the contour map is the presence of an elastic diffuse scattering intensity centered around the (001) Bragg peak that branches out along the (111) directions, and four weak satellite peaks which appear at specific locations along (111). A schematic indicating the location of the satellite features in reciprocal space which are incommensurate with the lattice is shown in Fig. 1(b). These peaks are most easily visualized by cuts along the (111) and (11) directions, as shown in the left panels of Fig. 2. Clearly, no superlattice reflections are present in the room temperature measurements (red symbols) indicating that they are not spurious in origin such as from multiple scattering. The order parameter, determined by measuring the temperature dependence of the intensity, was obtained for all three components visible in the contour map, the (001) ferromagnetic peak, the elastic diffuse, and the sat-
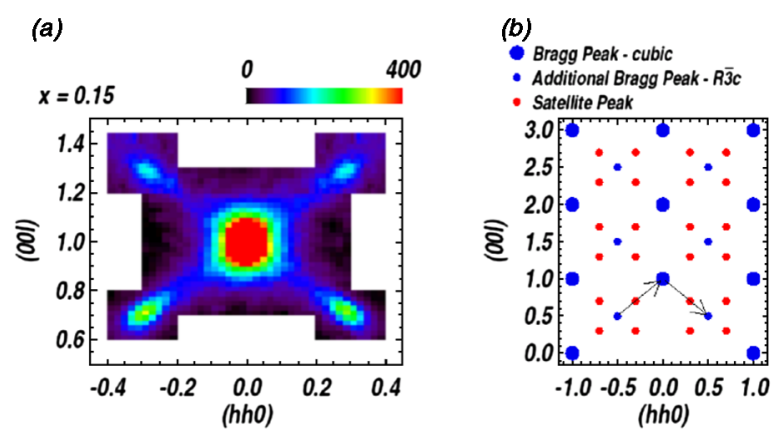

FIG. 1 (color online). (a) The elastic neutron scattering contour in the $(h h l)$ plane around (001) for $x=0.15$. Data were collected with an $E_{f}=3.6 \mathrm{meV}$, except for the top right corner that was measured with an $E_{f}=5.0 \mathrm{meV}$, and the top left corner is symmetrized with the top right corner. This peak originally consists of a nuclear and a magnetic component. The magnetic scattering is isolated by subtracting the spectrum collected at room temperature along with a constant background from data below $T_{c}$. Magnetic scattering appears only below the magnetic transition either to a spin glass or an FM phase. The broadening away from (001) is due to finite correlation length that is isotropically distributed suggesting the presence of ferromagnetic nanodroplets as discussed in Ref. [10]. The satellite peaks are visible along the (111) and (11) directions. (b) The positions of nuclear Bragg peaks and their four surrounding satellite peaks as they appear in the $h h l$ plane.

ellite peaks. The latter two features have the same order parameter, indicating that they must have the same origin. However, the long-range magnetic order for measurements very close to the (001) Bragg peak always increases prior to the appearance of the satellite peaks and the elastic diffuse scattering (right panels of Fig. 2) for all $x$. It is also the case that by measuring the satellite peak intensity as a function of momentum transfer, $Q$, we determined that since it follows the magnetic form factor it is magnetic in origin.

The appearance of a spin superstructure at a temperature lower than the macroscopic magnetic transition is quite surprising. The incommensurability $(\delta)$ that defines the modulation wave vector of the superlattice peaks as $(\delta$, $\delta, \delta$ ) is equal to $\vec{Q}_{\text {satellite }}-\vec{Q}_{\text {Bragg }}$. Scattering is observed at four incommensurate satellite positions, $(h \pm \delta, h \pm \delta$, $l \pm \delta$ ). $\delta$ exhibits a continuous dependence to $x$ as seen in Fig. 3(a). The ordering occurs along the (111) direction in the cubic notation which is a special axis in the rhombohedrally distorted crystal as this is the octahedral tilt direction leading to the rhombohedral symmetry ( $\mathrm{R} \overline{3} \mathrm{C})$. The correlation length, $\xi(\AA)$, of the satellite peaks is estimated in directions along and perpendicular to (111) from the widths of Lorentzian fits and found to be much broader than the instrumental resolution [Fig. 3(b)]. $\xi$ is indeed short for all $x$ which is not surprising because the real-space structure that give rise to this modulation is incommensurate with the lattice. It increases with $x$ and 


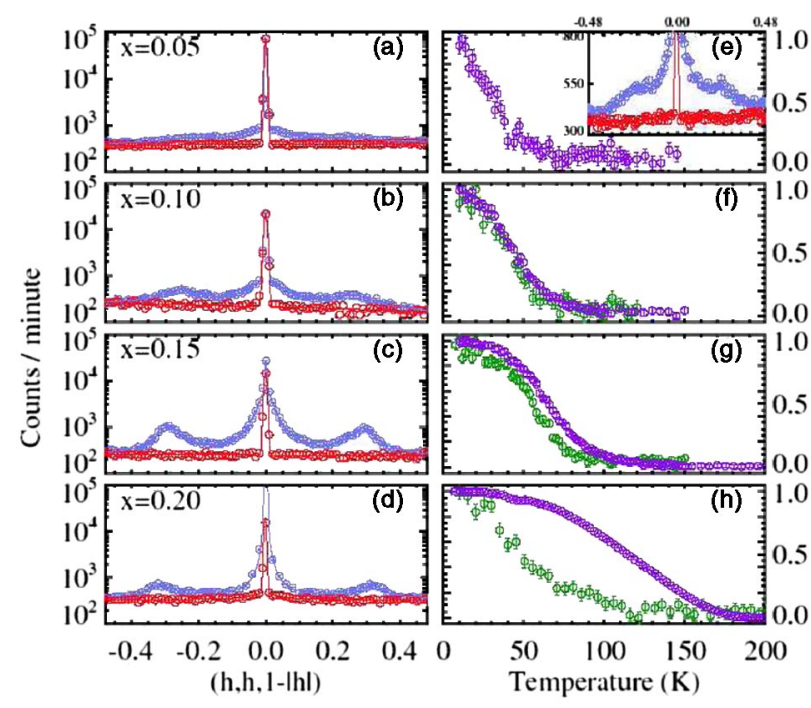

FIG. 2 (color online). The cuts along (111) and (111) are shown for (a) $x=0.05$, (b) 0.10 , (c) 0.15 , and (d) 0.20 . Also shown in the inset of (e) is an expanded cut for $x=0.05$. Measurements made above $T_{c}$ are shown in red, while those made at $8 \mathrm{~K}$ are shown in blue. Clear satellite peaks are present at low temperature and are absent at high temperature. The order parameter of the incommensurate peaks, depicted in green, and the ferromagnetic signal, depicted in purple for (e) $x=0.05$, (f) 0.10 , (g) 0.15 , and (h) 0.20 . The order parameter is subsequently rescaled to $0: 1$ by: $\left(I-I_{\min }\right) / I_{\max }$. The satellite peak intensity integrated along (111) is divided by the (001) peak intensity above $T_{c}$. The scattering is too weak in the case of $x=$ 0.05 to distinguish the order parameter of the satellite peak from the ferromagnetic scattering.

appears to level off at $x=0.15$. $\xi$ also varies with direction where it is longer in the perpendicular than in the parallel direction to (111). For instance, in $x=0.20$, the secondary spin ordering spans about two unit cells along (111) and about four unit cells along $(11 \overline{2})$ in cubic coordinates. Also shown in Fig. 3(b) is the integrated intensity of the satellite peaks that increases rapidly with doping up to $x=0.15$ $\left(x_{c}=0.18\right)$ but then drops substantially in the metallic phase at $x=0.20$. This suggests that the local order of the magnetic features that give rise to the incommensurate peaks is associated with the insulating phase, for $x<0.18$. However, some of this scattering remains at $x=0.20$ and might be directly competing with the charge mobility. For this reason, the integrated intensity is higher before the IMT but drops after IMT as the solid becomes metallic. Spin stripes in cuprates and nickelates also show incommensurate peaks that do not take on ideal fractional values. An explanation of the incommensurability from an ideal fraction could be a slight dislocation of the hole from the Co sites, which is reasonable considering that the Co ion hybridizes with the surrounding $\mathrm{O}$ ions and charge hopping between $\mathrm{Co}^{3+}$ and $\mathrm{Co}^{4+}$ sites is allowed even before IMT.

The fact that $\delta$ changes with $x$ suggests a connection of the magnetic modulation to the amount of charge doped

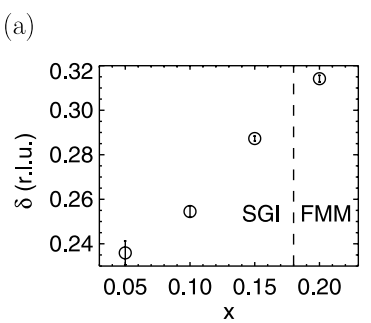

(b)

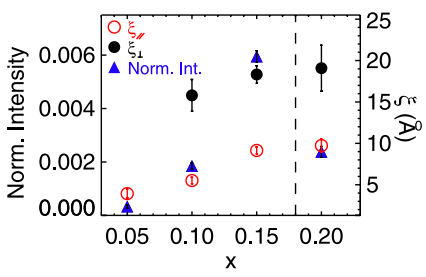

(c)

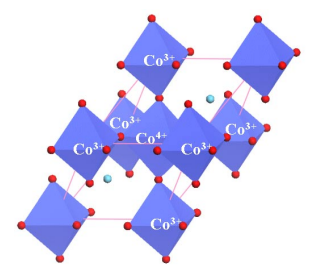

(d)

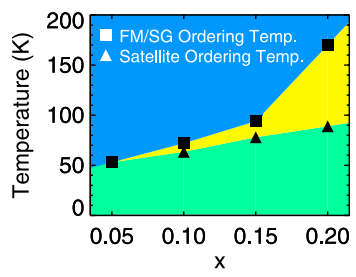

FIG. 3 (color online). (a) The incommensurate peak position, $\delta$, as a function of hole concentration. (b) The normalized satellite peak intensities and the correlation length, $\xi$, along and perpendicular to the (111) direction as a function of hole concentration. $\xi=1 / \gamma$, where $\gamma$ is the HWHM in momentum space. (c) A real-space illustration of the magnetopolarons made of $\mathrm{Co}^{3+}-\mathrm{Co}^{4+}$ clusters in the rhombohedral unit cell. Ordering of these units takes place in the (111) diagonal direction. (d) The phase diagram of $\mathrm{La}_{1-x} \mathrm{Sr}_{x} \mathrm{CoO}_{3}$ showing the two ordering temperatures. Blue indicates the paramagnetic regime where no magnetic ordering occurs. Yellow indicates the presence of only one magnetic phase. Green indicates the coexistence of the incommensurate, magnetic superstructure with the FM metallic clusters.

into the system, hence $\mathrm{Co}^{4+}$. One possibility arises from local ordering of $\mathrm{Co}^{3+}-\mathrm{Co}^{4+}$ ion clusters or extended magnetopolarons. For each $\mathrm{Co}^{4+}$ in the low spin state, six $\mathrm{Co}^{3+}$ in the intermediate spin configuration can be stabilized in the vicinity [17] forming a seven-site cluster. This is schematically depicted in Fig. 3(c). In this scenario, $\mathrm{Co}^{4+}$ is assumed to be a smaller ion than $\mathrm{Co}^{3+}$ allowing for the expansion of the $\mathrm{Co}^{3+}$ in the intermediate spin configuration $\left(t_{2 g}^{5} e_{g}^{1}\right)$. A similar argument is made in Ref. [18] but using the change in the size of the magnetic moments with $\mathrm{Sr}$ doping. The coupling within the extended magnetopolaron through double exchange lowers the kinetic energy of the charge. The charge interchange appears to be optimized when the clusters order along the (111) planes giving rise to $\mathrm{Co}^{4+}$-rich planes. The $\mathrm{Co}^{4+}$-rich planes form a periodic structure in the (111) direction squeezed between $\mathrm{Co}^{4+}$ - poor planes forming short-range stripes.

Our observations made by neutron scattering can be correlated to the detailed nature of the resistivity and magnetic susceptibility. Note that the macroscopic magnetic transition temperature does not vary significantly with the kind of divalent ion, $\mathrm{Ca}^{2+}, \mathrm{Sr}^{2+}$, or $\mathrm{Ba}^{2+}$ used at the $A$ site as a means for introducing charges into the system through the substitution of $\mathrm{La}^{3+}[17,18,20-22]$. In 
Ref. [23], the magnetic and transport characterization of the same crystals used in the current study is reported. Upon cooling, the resistivity, $\rho$, decreases in going through IMT, but saturates at low temperatures giving rise to a residual value. The residual resistivity in $\rho$ gets smaller with increasing $x$, and by $x=0.20, \rho$ becomes almost temperature independent after the transition [23,24]. Although the residual resistivity is small, $\sim 10^{-3} \Omega \mathrm{cm}$, it is higher than what is usually expected in metals [25] and puzzling, suggesting some kind of localization that reduces the effective number of carriers or their mobility in the metallic state. At the same time, one can estimate the percent change in the magnetoresistance and it is clear that it does not become "colossal". Combined with the present data, we can conclude that the onset of short-range spin and possibly charge correlations suppress metallicity and can explain the behavior in $\rho$. The observation of only a magnetic component to the ordering is not unusual as only a magnetic signal is also observed in the stripe ordering of $\mathrm{La}_{2-x} \mathrm{Sr}_{x} \mathrm{CuO}_{4}$ [26].

A new phase diagram that shows the composition dependence of the two ordering parameters is shown in Fig. 3(d). While the onset temperature of the incommensurability appears to increase linearly with $x, T_{c}$, on the other hand, deviates from such linearity particularly beyond the IMT $\left(x_{c}=0.18\right)$. The difference in the onset temperatures of the two order parameters is most noticeable for the $x=0.20$ which is a FM metal. The $\mathrm{Co}^{3+}{ }_{-} \mathrm{Co}^{4+}$ clusters bound together in a Zener-type polaron selforganize along the natural [111] rhombohedral axis, thereby driving the system to a more insulating state than otherwise expected. In the case of $x=0.20$, the FM metallic background is established at high temperatures and suppresses the secondary spin ordering with the result that it appears in a reduced form and at much lower temperatures. Doping even more charges and making the system more metallic should eventually lead to the disappearance of the secondary spin ordering altogether. This shows that metallic and insulating phases coexist where the suppression of one phase over the other is controlled by many parameters, one of which is charge kinetic energy. Towards the insulating phase, the incommensurate structures get stronger, while towards the metallic phase, the incommensurate structures get weaker.

To conclude, our neutron scattering data show a new phase suggesting that the phase diagram is more complex than previously thought in cobaltites. The local order of the proposed clusters is associated with the macroscopically insulating phase and it gets stronger with doping but decreases above the IMT. If the competition between the two order parameters is strong, phase separation between the magnetic itinerant and insulating, possibly charge- modulated, states could result. The presence of such unconventional states have played a major role in understanding the complexity of systems such as the manganites and cuprates and their observation in the cobaltites emphasizes their universal characteristic in this class of materials.

The authors would like to thank T. Egami, E. Dagotto, D. I. Khomskii, and J. M. Tranquada for useful discussions, and J.-H. Chung for help provided in some of the SPINS experiments. This work is supported by the U.S. Department of Energy under Contracts No. DE-FG0201ER45927 and No. DE-AC02-06CH11357, and the U.S. DOC through No. NIST-70NANB5H1152. The use of the neutron scattering facilities at NIST was supported in part through NSF Grants No. DMR-9986442 and No. DMR-0086210.

*Corresponding author.

Electronic address: louca@ virginia.edu

[1] S. Jin et al., Science 264, 413 (1994).

[2] E. Dagotto, Science 309, 257 (2005).

[3] J. M. Tranquada et al., Nature (London) 375, 561 (1995).

[4] K. Takada et al., Nature (London) 422, 53 (2003).

[5] M. A. Senaris-Rodrighez and J. B. Goodenough, J. Solid State Chem. 118, 323 (1995).

[6] S. Adler et al., Solid State Ionics 68, 193 (1994).

[7] Y. Tokura and N. Nagaosa, Science 288, 462 (2000).

[8] M. A. Korotin et al., Phys. Rev. B 54, 5309 (1996).

[9] T. Saitoh et al., Phys. Rev. B 56, 1290 (1997).

[10] D. Phelan et al., Phys. Rev. Lett. 96, 027201 (2006).

[11] D. Louca et al., Phys. Rev. B 60, R10 378 (1999).

[12] C. Zener, Phys. Rev. 81, 440 (1951).

[13] O. Zachar, S. A. Kivelson, and V. J. Emery, Phys. Rev. B 57, 1422 (1998).

[14] S. Mori, C. H. Chen, and S.-W. Cheong, Nature (London) 392, 473 (1998).

[15] J. B. Goodenough, Phys. Rev. 100, 564 (1955).

[16] A. Moreo, S. Yunoki, and E. Dagotto, Science 283, 2034 (1999).

[17] D. Louca and J.L. Sarrao, Phys. Rev. Lett. 91, 155501 (2003).

[18] V. Golovanov, L. Mihaly, and A. R. Moodenbaugh, Phys. Rev. B 53, 8207 (1996).

[19] M. Itoh et al., J. Phys. Soc. Jpn. 63, 1486 (1994).

[20] M. Kriener et al., Phys. Rev. B 69, 094417 (2004).

[21] J. Wu and C. Leighton, Phys. Rev. B 67, 174408 (2003).

[22] J. C. Burley, J. F. Mitchell, and S. Short, Phys. Rev. B 69, 054401 (2004).

[23] H. M. Aarbogh et al., Phys. Rev. B 74, 134408 (2006).

[24] Y. Onose and Y. Tokura, Phys. Rev. B 73, 174421 (2006).

[25] N. F. Mott and H. Jones, The Theory of the Properties of Metals and Alloys (Dover, New York, 1958), p. 277.

[26] M. Fujita et al., Phys. Rev. B 65, 064505 (2002). 